\title{
Lactobacilli Interfere with Streptococcus pyogenes Hemolytic Activity and Adherence to Host Epithelial Cells
}

\author{
Sunil D. Sarojt, Lisa Maudsdottert, Raquel Tavares and Ann-Beth Jonsson* \\ Department of Molecular Biosciences, The Wenner-Gren Institute, Stockholm University, Stockholm, Sweden
}

OPEN ACCESS

Edited by:

Victoria Louise Challinor

University of Copenhagen, Denmark

Reviewed by:

Atte Von Wright,

University of Eastern Finland, Finland

Raffaele Coppola,

University of Molise, Italy

*Correspondence:

Ann-Beth Jonsson

ann-beth.jonsson@su.se

tThese authors have contributed

equally to this work.

Specialty section:

This article was submitted to

Antimicrobials, Resistance

and Chemotherapy,

a section of the journal

Frontiers in Microbiology

Received: 15 June 2016

Accepted: 18 July 2016

Published: 29 July 2016

Citation:

Saroj SD, Maudsdotter L, Tavares $R$ and Jonsson A-B (2016) Lactobacilli Interfere with Streptococcus pyogenes Hemolytic Activity and Adherence to Host Epithelial Cells. Front. Microbiol. 7:1176. doi: 10.3389/fmicb.2016.01176
Streptococcus pyogenes [Group A streptococcus (GAS)], a frequent colonizer of the respiratory tract mucosal surface, causes a variety of human diseases, ranging from pharyngitis to the life-threatening streptococcal toxic shock-like syndrome. Lactobacilli have been demonstrated to colonize the respiratory tract. In this study, we investigated the interference of lactobacilli with the virulence phenotypes of GAS. The Lactobacillus strains L. rhamnosus Kx151A1 and L. reuteri PTA-5289, but not L. salivarius LMG9477, inhibited the hemolytic activity of $S$. pyogenes $S 165$. The inhibition of hemolytic activity was attributed to a decrease in the production of streptolysin S (SLS). Conditioned medium (CM) from the growth of $L$. rhamnosus $K \times 151 A 1$ and $L$. reuteri PTA-5289 was sufficient to down-regulate the expression of the sag operon, encoding SLS. The Lactobacillus strains L. rhamnosus Kx151A1, L. reuteri PTA-5289, and L. salivarius LMG9477 inhibited the initial adherence of GAS to host epithelial cells. Intriguingly, competition with a combination of Lactobacillus species reduced GAS adherence to host cells most efficiently. The data suggest that an effector molecule released from certain Lactobacillus strains attenuates the production of SLS at the transcriptional level and that combinations of Lactobacillus strains may protect the pharyngeal mucosa more efficiently from the initial colonization of GAS. The effector molecules released from Lactobacillus strains affecting the virulence phenotypes of pathogens hold potential in the development of a new generation of therapeutics.

Keywords: Streptococcus pyogenes, Lactobacillus, streptolysin, microbiota, adherence

\section{INTRODUCTION}

A range of bacterial communities including commensals and pathogens colonize the nasopharynx. The pharyngeal inhabitant Streptococcus pyogenes [group A streptococcus (GAS)], an adapted human pathogen, a common colonizer of the mucosa of the mouth, nose, and pharynx, is among the many pathogens that most often colonize their host asymptomatically and only occasionally cause disease. Local pharyngeal infection with GAS manifests as pharyngitis and, if spread from the local site, can cause the systemic diseases sepsis, streptococcal toxic shock syndrome, and necrotizing fasciitis (Luca-Harari et al., 2009). S. pyogenes produces a wide array of virulence factors, enabling it to adhere, invade, and spread within the human host (Cunningham, 2008). One of the characteristic features of $S$. pyogenes is the ability to lyse red blood cells (RBC), referred to as 
$\beta$-hemolysis. S. pyogenes produces two different hemolysins/ streptolysins, streptolysin S (SLS) and streptolysin O. The reason why $S$. pyogenes sometimes causes disease is not entirely understood, but both bacterial virulence factors and host factors are thought to contribute (Cole et al., 2011). The microbiota is one such host factor that needs further investigation. Attachment to epithelial cells is the crucial initial step of colonization because non-adherent GAS is removed by mucus and saliva flow. Additionally, bacterial interaction with the epithelial cells elicits multiple responses in the host cells, including cell signaling events, and modification of the host cell transcriptome (Nakagawa et al., 2004). The host responses regulate the bacterial colonization and play a significant role in the pathogenesis of the infection (Ribet and Cossart, 2015).

The microbiota prevents colonization with pathogenic bacteria and represents an important first line of defense. Mechanisms describing the probiotic effects of Lactobacillus strains include upregulation of mucin production in the host cells, interference with host pattern recognition receptors, competition for essential metabolites, production of antibacterial molecules, and co-aggregation between the bacteria of the microbiota and invading pathogenic bacteria, leading to interference with pathogen adherence to host cells (Lebeer et al., 2010; Reid et al., 2011; Lievin-Le Moal and Servin, 2014). Lactobacillus species are known to play a significant role in protection against many gastrointestinal and urogenital pathogens (Osset et al., 2001; Ostad et al., 2009). However, less is known about their antagonistic capacity against oralpharyngeal pathogens. Additionally, the detailed mechanisms behind the anti-adhesive properties of lactobacilli and their effect on the expression of virulence-associated genes are far from fully understood.

Different species of Lactobacillus are part of the microbiota of the mucosal membranes in the pharyngeal tract (Badet and Thebaud, 2008). The study, therefore, aimed to investigate whether Lactobacillus strains interfere with the expression of the virulence-associated factors of $S$. pyogenes and its ability to adhere to pharyngeal epithelial cells.

\section{MATERIALS AND METHODS}

\section{Bacterial Strains and Growth Conditions}

All Lactobacillus strains were isolated from healthy human individuals. Lactobacillus rhamnosus Kx151A1, isolated from a gastric biopsy, has been described previously (Roos et al., 2005). The Lactobacillus salivarius LMG9477, a type strain from the Belgian Coordinated Collections of Micro-organisms (BCCM), and Lactobacillus reuteri ATCC: PTA-5289, a kind gift from BioGaia AB, Stockholm were isolated from the oral cavity and have been described previously (de Klerk et al., 2016). Lactobacilli were grown on Rogosa agar plates and cultured in MRS broth (Oxoid, Thermo Fisher Scientific, Hampshire, UK). S. pyogenes S165 (GAS), serogroup emm6, was isolated from a septic patient (Sjolinder et al., 2008). GAS was grown on GC agar (Acumedia, Lansing, MI, USA) containing Kellogg's supplement
(Kellogg et al., 1963). All bacteria were cultured at $37^{\circ} \mathrm{C}$ and $5 \%$ $\mathrm{CO}_{2}$.

\section{Cell Lines and Culture Conditions}

The pharyngeal epithelial cell lines FaDu (ATCC: HTB-43), and Detroit 562 (ATCC: CCL-138) were cultured in DMEM with GlutaMAX and pyruvate (Invitrogen, Carlsbad, CA, USA) supplemented with $10 \%$ heat-inactivated fetal bovine serum (Sigma-Aldrich, St. Louis, MO, USA). The cells were maintained at $37^{\circ} \mathrm{C}$ and $5 \% \mathrm{CO}_{2}$ in a humidified environment. In all experiments, epithelial cells were seeded into cell culture plates the day before experiments to reach $90 \%$ confluence. The cell medium was replaced with serum-free DMEM half an hour before the experiments.

\section{Conditioned Media (CM)}

Lactobacilli were grown on Rogosa agar plates. The bacteria were scraped off, washed twice with Todd-Hewitt broth (THB), and resuspended in THB. From this suspension, the growth of lactobacilli was initiated by adjusting to $\mathrm{A}_{600} \approx 0.1$ in THB. The growth was monitored until the density reached $\mathrm{A}_{600} \approx 1.0$. The lactobacilli were removed by centrifugation. The supernatant was passed through a $0.2 \mu \mathrm{m}$ filter, adjusted to $\mathrm{pH} 7.8$ and supplemented with $2 \%$ tryptone to obtain the Conditioned Media (CM, Saroj and Rather, 2013).

\section{Hemolytic Activity}

Group A streptococcus was cultured in THB, co-incubated with lactobacilli, or in CM. For co-incubation, GAS and lactobacilli from agar plates were suspended in $\mathrm{THB}$ at $\mathrm{A}_{600}=0.1$ in the proportion of $1: 1$. At the desired time point, the bacteria were harvested by centrifugation; the supernatants were passed through a $0.2 \mu \mathrm{m}$ filter and used for the hemolytic assay. RBCs for hemolytic assays were prepared from horse blood (Håtunalab $\mathrm{AB}$, defibrinated) by washing three times in phosphate buffered saline (PBS) and diluting to $2 \%$ in PBS. The supernatants were diluted 1:10, mixed 1:1 with RBCs and incubated at $37^{\circ} \mathrm{C}$ in $5 \% \mathrm{CO}_{2}$ for $1 \mathrm{~h}$. The RBCs were removed by centrifugation at $2000 \mathrm{~g}$ for $3 \mathrm{~min}$, and the supernatant was analyzed at $\mathrm{A}_{404}$ for hemolysis. Hemolytic activity was reported as the percentage relative to water alone. The SLSmediated hemolytic activity was confirmed using trypan blue and cholesterol binding assays as SLS gets inhibited in the presence of trypan blue and SLO by cholesterol (Sierig et al., 2003). For all assays, the hemolytic activity was examined in the presence of trypan blue and cholesterol. The hemolytic activity of GAS in lactobacilli supernatants was measured by harvesting the supernatants at $1 \mathrm{~h}$ intervals until the growth reached the stationary phase. For hemolytic activity of GAS supernatant in co-incubation with $\mathrm{CM}$, supernatant from the growth of GAS in THB from $\mathrm{A}_{600} \approx 0.1$ to $\mathrm{A}_{600} \approx 1.0$ was obtained. The supernatant was mixed with THB, or with CM from L. salivarius (L.sa), L. rhamnosus (L.rh), or L. reuteri (L.re) in a proportion of $1: 1$ and incubated for 30 min at $37^{\circ} \mathrm{C}$ with $5 \% \mathrm{CO}_{2}$ prior to measuring the hemolytic activity. 


\section{Viable Count}

To asses the viability of GAS, viable count was performed by serial dilutions and plating on GC agar. In co-incubation experiments, the bacteria could be distinguished by colony size; GAS formed colonies larger than lactobacilli.

\section{Quantitative PCR Assays}

Group A streptococcus was grown alone or co-incubated with lactobacilli in $\mathrm{THB}$ or in $\mathrm{CM}$ to $\mathrm{A}_{600} \approx 1.0$. The cells were harvested by centrifugation and total RNA was isolated using an RNeasy mini kit (Qiagen) after treatment with mutanolysin for $1 \mathrm{~h}$. Total RNA $200 \mathrm{ng}$ was reverse transcribed to cDNA using SuperScript VILO ${ }^{\mathrm{TM}}$ master mix (ThermoFisher Scientific). The primers sagA-RT-(F) 5'GCTACTAGTGTAGCTGAAACAACT CAA-3' and sagA-RT-(R) 5'AGCAACAAGTAGTACAGCAGC AA- $3^{\prime}$ were used for the qPCR. The following conditions were used: an initial denaturation step at $95^{\circ} \mathrm{C}$ for $10 \mathrm{~min}$; 45 cycles at $95^{\circ} \mathrm{C}$ for $15 \mathrm{~s}$ and $60^{\circ} \mathrm{C}$ for $1 \mathrm{~min}$. The fold change was measured in relation to the housekeeping gene gyrA using the primers gyrA-RT-(F) 5'CGACTT GTCTGAACGCCAAAGT3' and gyrART-(R) 5'ATCACGTTCCAAACCAGTCAAAC3'.

\section{Luciferase Assay}

The transcriptional fusion for the luciferase assay was prepared using pKSM720, which encodes a promoterless firefly luciferase (Almengor et al., 2007). The 400 bp region upstream of the sag operon was amplified using the primers sagAPro-F- 5'CCTGAGGATCCGTATTAGCGAGGTA AAG3 ${ }^{\prime}$ and sagAPro-R- 5' AGTCACTCGAGAAGGTTTACCTCC TTATC3'. The fragments were gel purified, digested with BamHI and XhoI and cloned into the BglII and XhoI site of pKSM720. The resulting construct, pKSM720-sagApro-luc, was electroporated into GAS. Spectinomycin at $50 \mu \mathrm{g} / \mathrm{ml}$ was used to maintain the plasmid. The GAS with the construct was grown in THB or CM from lactobacilli from $\mathrm{A}_{600} \approx 0.1$ to $\mathrm{A}_{600} \approx 1.0$, and the luciferase activity measured according to the manufacturer's instructions (Promega). The assay was conducted in the absence of spectinomycin. The maintenance of the plasmid construct was confirmed from the viable counts on THY plates with or without spectinomycin.

\section{Adherence Assays}

Adherence assays were performed as previously described (Maudsdotter et al., 2011). FaDu (ATCC: HTB-43) or Detroit 562 (ATCC: CCL-138) pharyngeal epithelial cells were cultured in a 48-well plate and were inoculated with GAS at an MOI of 100 or together with lactobacilli at an MOI of 100 for $2 \mathrm{~h}$. In competition assays, GAS and lactobacilli were added simultaneously. In exclusion assays, lactobacilli were preincubated with epithelial cells for $2 \mathrm{~h}$ followed by washing to remove unbound bacteria before the addition of GAS. In displacement assays, GAS was pre-incubated with the epithelial cells for $2 \mathrm{~h}$ followed by washing before addition of lactobacilli. When mixtures of lactobacilli were used, an MOI of 100 for each strain was added to the epithelial cells. After incubation, unbound bacteria were removed by washing in the medium.
Adhered bacteria were quantified from viable counts obtained by lysing the epithelial cells for $5 \mathrm{~min}$ in $1 \%$ saponin and plating on GC plates. The adherence of GAS alone was normalized to 1 .

\section{Statistical Analysis}

All the experiments were performed in triplicate and repeated three times. Analysis of variance (ANOVA) and Tukey's HSD (honestly significantly different) test (Statistica) was used to analyze differences between the groups for statistical significance. Statistical analysis of ratios or relative values was performed on log ratios. A $p$-value below 0.05 was considered statistically significant. Error bars represent standard deviation.

\section{RESULTS}

\section{Certain Lactobacilli Inhibit the Hemolytic Activity of S. pyogenes}

We investigated the influence of lactobacilli on the hemolytic activity of GAS. GAS was co-incubated with different Lactobacillus strains in THB and assayed for hemolytic activity. L. rhamnosus Kx151A1 (L. rh) and L. reuteri PTA-5289 (L. re) inhibited the hemolytic activity of GAS (Figure 1A). However, L. salivarius LMG9477 (L. sa) had no effect. The hemolytic activity was mediated by SLS because the presence of trypan blue, but not cholesterol, blocked the hemolytic activity (data not shown). To rule out the possibility that lactobacilli may inhibit the growth of GAS or affect the $\mathrm{pH}$, we performed viable counts and measured the $\mathrm{pH}$ in the medium. The incubation of GAS with lactobacilli did not affect the survival of GAS (Figure 1B). Additionally, the $\mathrm{pH}$ of the growth media was not significantly affected during the co-incubation (Figure 1C). These results show that certain lactobacilli block the SLS-mediated hemolytic activity of GAS.

\section{Lactobacillus Growth Supernatant Inhibits the Hemolytic Activity of \\ S. pyogenes}

To examine whether the inhibition of hemolytic activity was due to molecules released from lactobacilli, a conditioned medium (CM) was prepared from the growth supernatants of lactobacilli. The hemolytic activity of GAS in the CM was assessed at regular intervals. The SLS-mediated hemolytic activity was inhibited in GAS grown in the CM from L. rhamnosus (L. rh) and L. reuteri (L. re) throughout the growth period (Figure 2A). As previously reported, hemolytic activity was detected during the logarithmic phase and reached a maximum in the stationary phase (Kinkel and McIver, 2008). The growth of GAS was not affected in the CM (Figure 2B). These results indicate that the inhibitory molecule is a component released from lactobacilli.

To examine whether the released effector molecule binds directly to SLS, supernatants from the growth of GAS in THB were mixed with the CM of lactobacilli and assayed for hemolytic activity. There was no observable difference in the hemolytic activity of the GAS supernatant mixed with fresh THB and the GAS supernatant mixed with CM from lactobacilli (Figure 2C). 
A

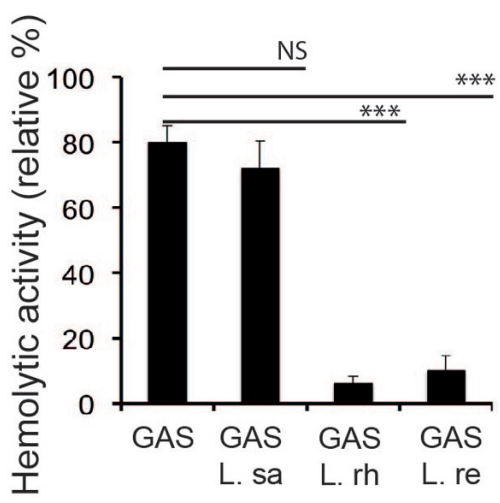

B

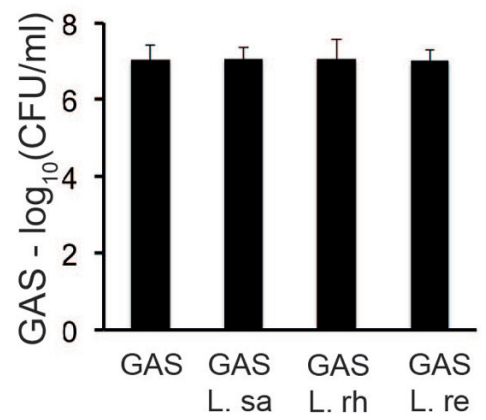

C

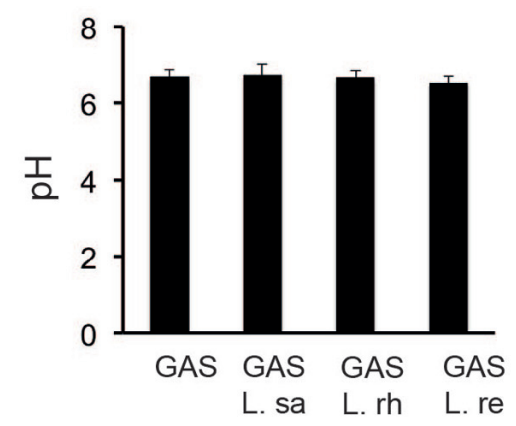

FIGURE 1 | Lactobacilli inhibit the hemolytic activity of Group A streptococcus (GAS). (A) Hemolytic activity of GAS grown alone or co-incubated with Lactobacillus rhamnosus (L. rh), L. reuteri (L. re), or L. salivarius (L. sa) in THB. (B) Viable count of GAS (C) pH of the supernatant from the growth of GAS in THB alone or co-incubated with lactobacilli. Significant differences are marked with asterisks.

A

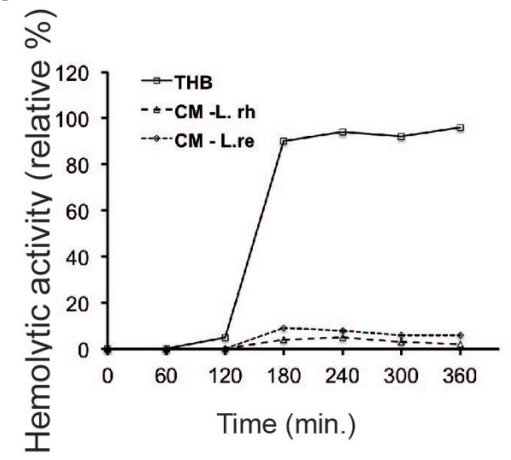

B

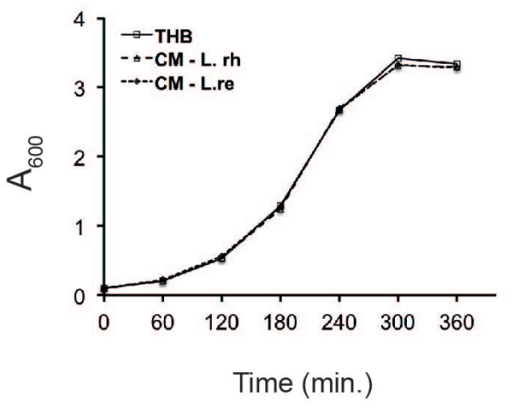

C

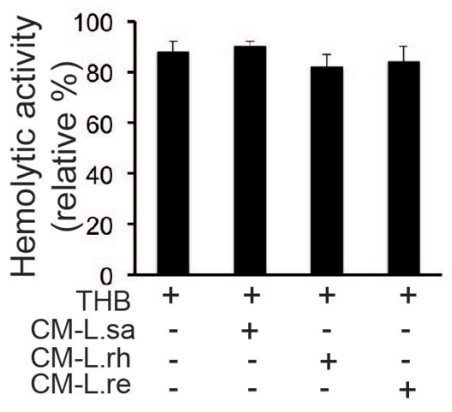

FIGURE 2 | Lactobacillus growth supernatant inhibits the hemolytic activity of GAS (A) Hemolytic activity of GAS grown in THB, CM of L. rhamnosus (L. rh) and L. reuteri (L. re). (B) Growth curve of GAS in THB and CM. (C) Hemolytic activity of GAS supernatant in co-incubation with CM. The supernatant obtained from the growth of GAS in THB was mixed with CM.

These data suggest that Lactobacillus strains release or secrete a soluble effector molecule that inhibits the hemolytic activity of GAS.

\section{Lactobacilli Interfere with the Expression of the sag Operon}

Streptolysin S is chromosomally encoded by a contiguous ninegene sag operon (Borgia et al., 1997; Molloy et al., 2011). The first gene of the operon is $\operatorname{sag} A$, which encodes a 53 amino acid precursor. The expression of sagA during the coincubation of GAS with lactobacilli was monitored by $\mathrm{qPCR}$. There was no significant $(P \geq 0.05)$ change in the expression of sagA when GAS was co-incubated with L. salivarius (L. sa) (Figure 3A). However, when GAS was co-incubated with cultures of L. rhamnosus (L. rh) and L. reuteri (L. re), a significant $(P<0.01)$ decrease in sagA expression was observed. A similar effect was observed in the expression of sag A with GAS cultured in the CM of lactobacilli (Figure 3B). CM from L. rhamnosus (L. rh) and L. reuteri (L. re), but not
L. salivarius (L. sa), inhibited sagA expression. The results indicate that the decrease in the hemolytic activity of GAS by lactobacilli is due to a decrease in the expression of the sag operon.

\section{Lactobacilli Interfere with the Promoter Activity of the sag Operon}

To confirm that the effector molecule released from Lactobacillus inhibits the SLS at the transcriptional level, we constructed a transcriptional fusion of the promoter region of the sag operon to a promoterless luciferase reporter gene, sagApro-luc. GAS harboring sagApro-luc exhibited the expected luciferase activity when grown in THB. However, the luciferase activity was inhibited when the GAS with sagApro-luc was cultured in the CM from L. rhamnosus (L. rh) and L. reuteri (L. re) (Figure 4), whereas the CM of L. salivarius (L. sa) had no effect. The data demonstrate that a lactobacilli-released effector molecule regulates the GAS SLS-mediated hemolytic activity at the transcriptional level. 
A

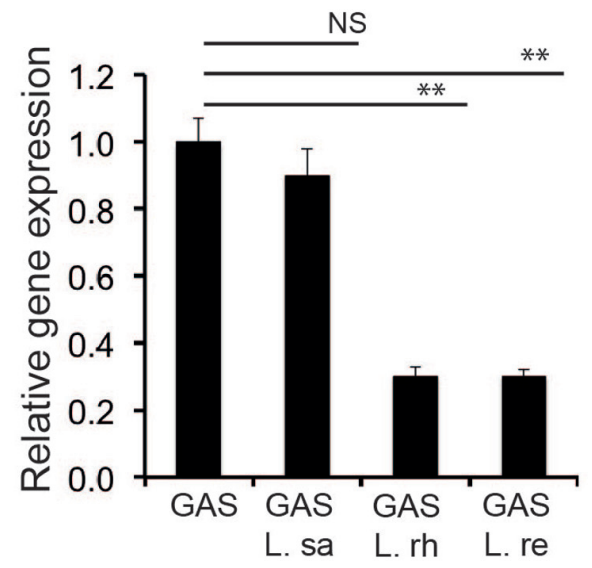

B

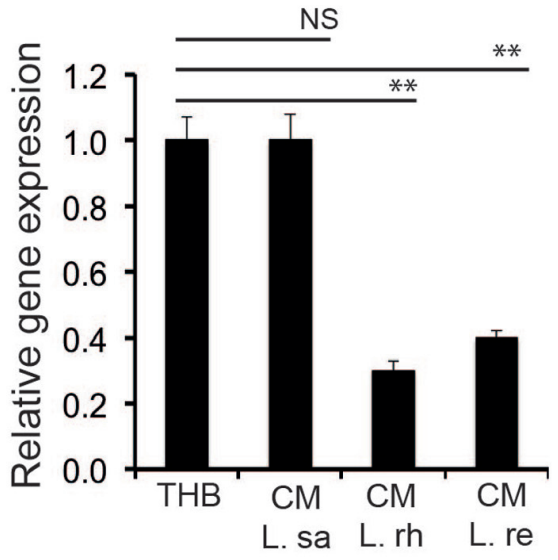

FIGURE 3 | Lactobacilli interfere with the expression of sagA. (A) Expression of sagA during co-incubation of GAS with lactobacilli (B) Influence of the Lactobacillus effector molecule in CM on the expression of sagA. Significant differences are marked with asterisks.

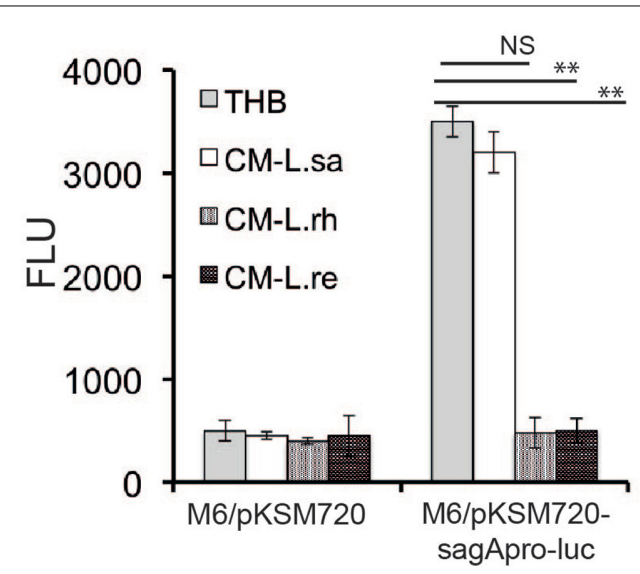

FIGURE 4 | Lactobacilli released component affects the promoter activity of sagA. A luciferase assay was performed to determine the effect of the Lactobacillus-released effector molecule on the promoter activity of sagA. Significant differences are marked with asterisks.

\section{Lactobacilli Inhibit the Adherence of GAS to Host Epithelial Cells}

In addition to their effect on hemolytic activity, we also investigated if lactobacilli have an effect on the initial adherence of GAS to pharyngeal epithelial cells. All the Lactobacillus isolates, i.e., L. rhamnosus (L. rh), L. reuteri (L. re), and L. salivarius (L. sa), significantly $(P \leq 0.01)$ reduced the adherence of GAS by approximately $35 \%$ in a competition assay (Figure 5A). Additionally, all three Lactobacillus isolates significantly $(P \leq 0.01)$ inhibited streptococcal adherence when pre-incubated with the host cells for $2 \mathrm{~h}$ before the addition of GAS in exclusion assays. However, in the exclusion assays, a difference between the Lactobacillus isolates was observed: L. rhamnosus and L. reuteri inhibited streptococcal adherence by approximately $35 \%$, whereas approximately $20 \%$ inhibition was observed with $L$. salivarius. Additionally, the ability of lactobacilli to displace already-adhered GAS was tested. L. salivarius significantly $(P \leq 0.01)$ inhibited streptococcal adherence by $35 \%$ in displacement assays. In contrast, neither $L$. rhamnosus nor L. reuteri displaced adhered GAS. In a previous study, we showed that co-incubating GAS with lactobacilli for longer time points (4-16 h) reduced GAS viability and thereby reduced the number of viable GAS adhered to host cells (Maudsdotter et al., 2011). However, co-incubating host cells with GAS and lactobacilli for $2 \mathrm{~h}$ did not affect streptococcal viability (Figure 5B). These data show that lactobacilli significantly reduce GAS adherence to epithelial cells.

\section{Effect of Different Combinations of Lactobacillus Strains on the Adherence of GAS}

Different combinations of Lactobacillus isolates were coincubated with GAS to evaluate whether a combination of lactobacilli enhanced the anti-adhesive effect. A combination of L. rhamnosus (L. rh) or L. reuteri (L. re) with L. salivarius (L. sa) conferred a significantly $(P \leq 0.01)$ higher level of inhibition compared to co-incubation with any of the Lactobacillus isolates alone (Figure 6A). However, a combination of L. rhamnosus (L. rh) and L. reuteri (L. re) did not enhance the anti-adhesive effect compared to each species alone (Figure 6A). The effect of different combinations of lactobacilli on GAS adherence was tested in an additional cell line, pharyngeal epithelial Detroit 562 cells, and the same effects were observed (Figure 6B). Thus, a combination of Lactobacillus strains can increase the antiadhesive effect.

\section{DISCUSSION}

Group A streptococcus and lactobacilli are both part of the pharyngeal microbiota. This study aimed to investigate 
A

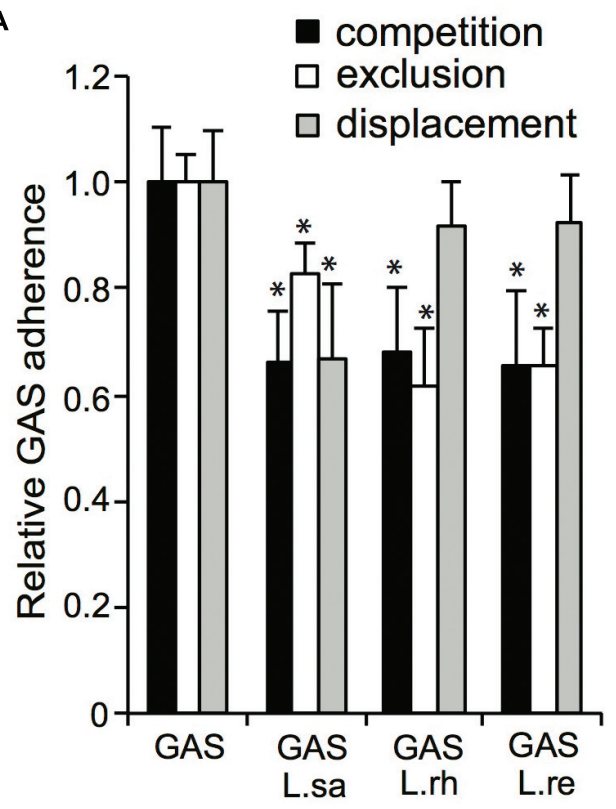

B

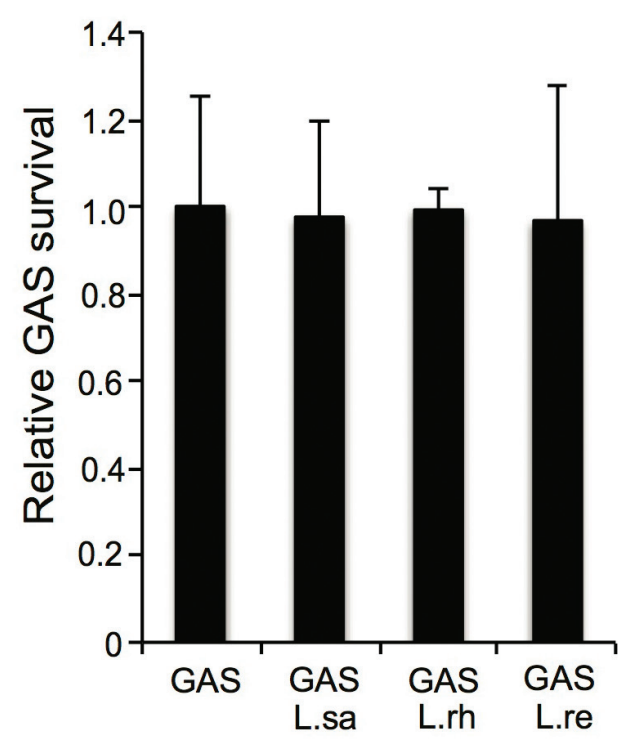

FIGURE 5 | Lactobacilli inhibit GAS adherence to pharyngeal epithelial host cells. (A) Adherence of GAS to FaDu epithelial cells in a competition, exclusion and displacement assay (B) Recovery of GAS after co-infection with lactobacilli for $2 \mathrm{~h}$. Significant differences are marked with an asterisk.

A

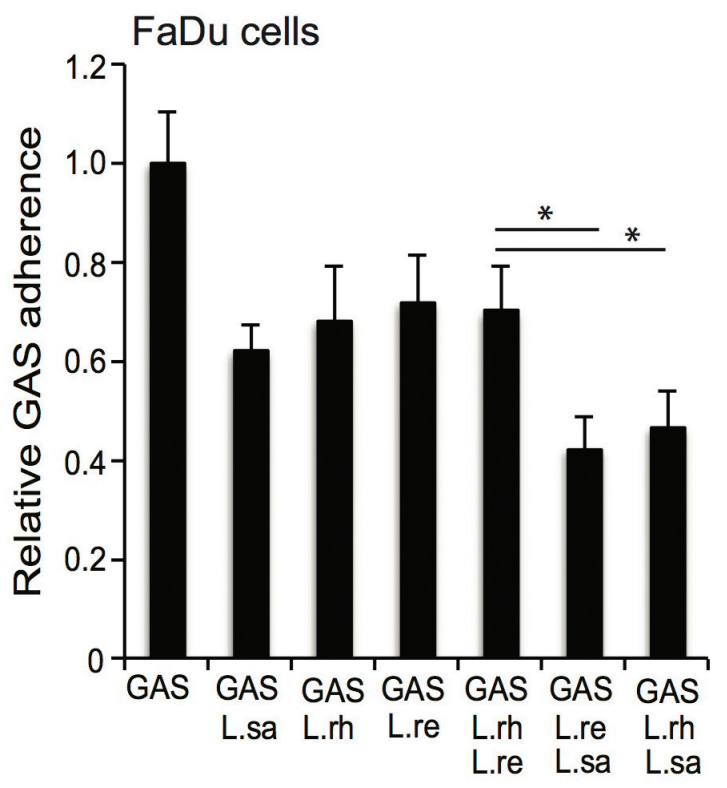

B

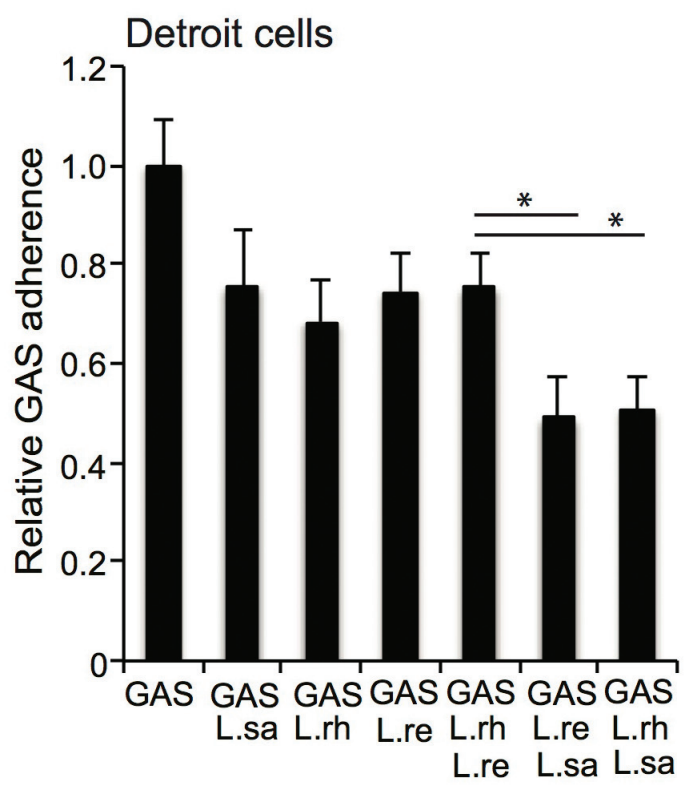

FIGURE 6 | Group A streptococcus adherence to epithelial cells in competition with different combinations of lactobacilli. GAS adherence to (A) FaDu cells and (B) Detroit 562 cells after infection alone or together with L. salivarius (L.sa), L. rhamnosus (L.rh), and L. reuteri (L.re) for 2 h. Significant differences are marked with an asterisk.

how lactobacilli affect the virulence phenotypes of GAS in terms of hemolytic activity and adherence to host epithelial cells.

Lactobacilli are a heterogeneous group of bacteria. Additionally, it has been previously reported that different lactobacillus isolates vary widely in their ability to interfere with the virulence mechanisms of pathogens (Osset et al., 2001; Ekmekci et al., 2009; Ostad et al., 2009). GAS infections have been attributed to the variety of secreted and surface-bound virulence factors. One of the most important virulence factors 
encoded by GAS is SLS (Ginsburg, 1999). In addition to the ability to lyse RBCs, SLS has been shown to damage the cell membranes of lymphocytes, neutrophils, platelets, lysosomes, and mitochondria, indicating an important role for SLS in S. pyogenes pathogenesis (Ginsburg, 1999; Molloy et al., 2011). In this study, we found that lactobacilli were able to inhibit the hemolytic activity of GAS. Moreover, CM from the growth of lactobacilli was sufficient to inhibit SLS production. The transcriptional fusion and $\mathrm{qPCR}$ data revealed that this effect takes place at the transcriptional level. The secreted components from lactobacilli negatively regulate the promoter activity and down-regulate the sag operon.

Incoming pathogens must compete with the resident microbiota for nutrients and space to colonize the mucosal membranes in the body. Additionally, the microbiota modulate immune responses, produce inhibitory substances and prevent the attachment of pathogens and in this way protect the host from infections (Lebeer et al., 2010). Lactobacilli of different species belong to the microbiota of the pharynx, the gastric tract, and the urogenital tract and inhibit the adherence of many pathogens (Alexandre et al., 2014; Ribet and Cossart, 2015). In a few studies, the Lactobacillus components mediating adherence inhibition have been identified; lactobacilli produce biosurfactants (Servin, 2004; Rizzo et al., 2013), S-layer proteins (Velraeds et al., 1996, 1998; Spurbeck and Arvidson, 2010), and surface-located enolase (Ren et al., 2012), which have been reported to inhibit the adherence of different pathogens. However, the mechanisms by which Lactobacillus species inhibit the adherence of pathogens are still largely unknown. Some studies have reported a similar level of adherence inhibition when lactobacilli are added to host cells together with the pathogen as that observed when lactobacilli pre-colonize the host cells (Lee et al., 2003; Zhang et al., 2010). Other studies have reported a stronger inhibition of lactobacilli pre-colonizing the host cells when compared to lactobacilli added simultaneously with the pathogen (Vielfort et al., 2008). In this study, we demonstrate that $L$. rhamnosus Kx151A1, L. reuteri PTA5289, and L. salivarius LMG9477 reduced the adherence of GAS to the host epithelial cells. In addition, we observed that L. salivarius conferred stronger protection when added in competition with GAS compared to when it precolonized the host cells. Further, L. salivarius was the only Lactobacillus species with the ability to displace alreadyadhered GAS. Our results highlight the differences between Lactobacillus species in the mechanism by which they confer colonization resistance as well as the effects they exert on host cells.

Various Lactobacillus species have previously been found to exhibit bactericidal activities against GAS in co-infection studies. In a previous study, we showed that co-incubation with lactobacilli for longer time points caused elevated lactic acid concentrations that are bactericidal against GAS and thereby reduce the number of viable GAS adhered to host cells (Almengor et al., 2007). In this study, we investigated the influence of lactobacilli on GAS adherence at a shorter time point at which GAS was not killed and observed inhibition of the initial adherence that was independent of compromised viability.

Thus, colonization with Lactobacillus species impairs GAS pathogenicity at different stages of infection, both by reducing the adherence at earlier stages and by affecting the viability after prolonged incubation and attenuating the hemolytic activity.

Probiotics are live microorganisms that confer health benefits on the host. Lactobacillus is a well-studied genus for probiotic use. Many criteria have been suggested for the selection of probiotics, including their ability to prevent the adherence of pathogens to target cells (Lee et al., 2003; Lebeer et al., 2010). The effects of probiotics are strain-specific, and the mechanisms of protection are largely unknown. The current study reveals that lactobacilli interfere with the virulence phenotypes of GAS in terms of SLS production and adherence to the host epithelial cells. Furthermore, a combination of lactobacilli has a greater effect in reducing the adherence of GAS to host epithelial cells and thus has an enhanced protective effect.

The findings may have a potential application in the use of lactobacilli strains as a probiotic for oral health purposes to combat GAS infections. Also, the secreted component from the lactobacilli holds a therapeutic value to fight GAS infections.

The identification of the Lactobacillus-secreted product interfering with the transcription of the sag operon is under study. Greater understanding of the molecular mechanisms by which probiotics function will help in developing enhanced future therapeutics.

\section{AUTHOR CONTRIBUTIONS}

Conceived and designed the experiments: SS, LM, A-BJ. Performed experiments: SS, LM, RT. Analyzed the data and wrote manuscript: SS, LM, A-BJ.

\section{FUNDING}

This work was supported by the Swedish Research Council, the Swedish Cancer Society, Knut and Alice Wallenbergs Stiftelse, and Torsten and Ragnar Söderbergs Stiftelse.

\section{ACKNOWLEDGMENTS}

We thank Prof. Kevin S. McIver, University of Maryland for providing pKSM720. We thank Nazeer Kanakryyh for his technical assistance. 


\section{REFERENCES}

Alexandre, Y., Le Berre, R., Barbier, G., and Le Blay, G. (2014). Screening of Lactobacillus spp. for the prevention of Pseudomonas aeruginosa pulmonary infections. BMC Microbiol. 14:107. doi: 10.1186/1471-2180-14-107

Almengor, A. C., Kinkel, T. L., Day, S. J., and McIver, K. S. (2007). The catabolite control protein CcpA binds to Pmga and influences expression of the virulence regulator Mga in the Group A Streptococcus. J. Bacteriol. 189, 8405-8416. doi: 10.1128/JB.01038-07

Badet, C., and Thebaud, N. B. (2008). Ecology of lactobacilli in the oral cavity: a review of literature. Open Microbiol. J. 2, 38-48. doi: $10.2174 / 1874285800802010038$

Borgia, S. M., Betschel, S., Low, D. E., and de Azavedo, J. C. (1997). Cloning of a chromosomal region responsible for streptolysin S production in Streptococcus pyogenes. Adv. Exp. Med. Biol. 418, 733-736. doi: 10.1007/978-1-4899-18253_172

Cole, J. N., Barnett, T. C., Nizet, V., and Walker, M. J. (2011). Molecular insight into invasive group A streptococcal disease. Nat. Rev. Microbiol. 9, 724-736. doi: $10.1038 /$ nrmicro2648

Cunningham, M. W. (2008). Pathogenesis of group A streptococcal infections and their sequelae. Adv. Exp. Med. Biol. 609, 29-42. doi: 10.1007/978-0-38773960-1_3

de Klerk, N., Maudsdotter, L., Gebreegziabher, H., Saroj, S. D., Eriksson, B., Eriksson, O. S., et al. (2016). Lactobacilli reduce Helicobacter pylori attachment to host gastric epithelial cells by inhibiting adhesion gene expression. Infect. Immun. 84, 1526-1535. doi: 10.1128/IAI.00163-16

Ekmekci, H., Aslim, B., and Ozturk, S. (2009). Characterization of vaginal lactobacilli coaggregation ability with Escherichia coli. Microbiol. Immunol. 53, 59-65. doi: 10.1111/j.1348-0421.2009.00115.x

Ginsburg, I. (1999). Is streptolysin S of group A streptococci a virulence factor? APMIS 107, 1051-1059. doi: 10.1111/j.1699-0463.1999.tb01509.x

Kellogg, D. S. Jr., Peacock, W. L. Jr., Deacon, W. E., Brown, L., and Pirkle, D. I. (1963). Neisseria gonorrhoeae. I. virulence genetically linked to clonal variation. J. Bacteriol. 85, 1274-1279.

Kinkel, T. L., and McIver, K. S. (2008). CcpA-mediated repression of streptolysin $\mathrm{S}$ expression and virulence in the group A Streptococcus. Infect. Immun. 76, 3451-3463. doi: 10.1128/IAI.00343-08

Lebeer, S., Vanderleyden, J., and De Keersmaecker, S. C. (2010). Host interactions of probiotic bacterial surface molecules: comparison with commensals and pathogens. Nat. Rev. Microbiol. 8, 171-184. doi: 10.1038/nrmicro2297

Lee, Y. K., Puong, K. Y., Ouwehand, A. C., and Salminen, S. (2003). Displacement of bacterial pathogens from mucus and Caco-2 cell surface by lactobacilli. J. Med. Microbiol. 52(Pt 10), 925-930. doi: 10.1099/jmm.0.05009-0

Lievin-Le Moal, V., and Servin, A. L. (2014). Anti-infective activities of Lactobacillus strains in the human intestinal microbiota: from probiotics to gastrointestinal anti-infectious biotherapeutic agents. Clin. Microbiol. Rev. 27, 167-199. doi: 10.1128/CMR.00080-13

Luca-Harari, B., Darenberg, J., Neal, S., Siljander, T., Strakova, L., Tanna, A., et al. (2009). Clinical and microbiological characteristics of severe Streptococcus pyogenes disease in Europe. J. Clin. Microbiol. 47, 1155-1165. doi: 10.1128/JCM.02155-08

Maudsdotter, L., Jonsson, H., Roos, S., and Jonsson, A. B. (2011). Lactobacilli reduce cell cytotoxicity caused by Streptococcus pyogenes by producing lactic acid that degrades the toxic component lipoteichoic acid. Antimicrob. Agents Chemother. 55, 1622-1628. doi: 10.1128/AAC.00770-10

Molloy, E. M., Cotter, P. D., Hill, C., Mitchell, D. A., and Ross, R. P. (2011). Streptolysin S-like virulence factors: the continuing sagA. Nat. Rev. Microbiol. 9, 670-681. doi: 10.1038/nrmicro2624

Nakagawa, I., Nakata, M., Kawabata, S., and Hamada, S. (2004). Transcriptome analysis and gene expression profiles of early apoptosis-related genes in Streptococcus pyogenes-infected epithelial cells. Cell. Microbiol. 6, 939-952. doi: 10.1111/j.1462-5822.2004.00412.x

Osset, J., Bartolome, R. M., Garcia, E., and Andreu, A. (2001). Assessment of the capacity of Lactobacillus to inhibit the growth of uropathogens and block their adhesion to vaginal epithelial cells. J. Infect. Dis. 183, 485-491. doi: $10.1086 / 318070$
Ostad, S. N., Salarian, A. A., Ghahramani, M. H., Fazeli, M. R., Samadi, N., and Jamalifar, H. (2009). Live and heat-inactivated lactobacilli from feces inhibit Salmonella Typhi and Escherichia coli adherence to Caco-2 cells. Folia Microbiol. (Praha) 54, 157-160. doi: 10.1007/s12223-009-0024-7

Reid, G., Younes, J. A., Van der Mei, H. C., Gloor, G. B., Knight, R., and Busscher, H. J. (2011). Microbiota restoration: natural and supplemented recovery of human microbial communities. Nat. Rev. Microbiol. 9, 27-38. doi: 10.1038/nrmicro2473

Ren, D., Li, C., Qin, Y., Yin, R., Li, X., Tian, M., et al. (2012). Inhibition of Staphylococcus aureus adherence to Caco-2 cells by lactobacilli and cell surface properties that influence attachment. Anaerobe 18, 508-515. doi: 10.1016/j.anaerobe.2012.08.001

Ribet, D., and Cossart, P. (2015). How bacterial pathogens colonize their hosts and invade deeper tissues. Microbes Infect. 17, 173-183. doi: 10.1016/j.micinf.2015.01.004

Rizzo, A., Losacco, A., Carratelli, C. R., Domenico, M. D., and Bevilacqua, N. (2013). Lactobacillus plantarum reduces Streptococcus pyogenes virulence by modulating the IL-17, IL-23 and Toll-like receptor $2 / 4$ expressions in human epithelial cells. Int. Immunopharmacol. 17, 453-461. doi: 10.1016/j.intimp.2013.07.005

Roos, S., Engstrand, L., and Jonsson, H. (2005). Lactobacillus gastricus sp. nov., Lactobacillus antri sp. nov., Lactobacillus kalixensis sp. nov. and Lactobacillus ultunensis sp. nov., isolated from human stomach mucosa. Int. J. Syst. Evol. Microbiol. 55(Pt 1), 77-82. doi: 10.1099/ijs.0.63083-0

Saroj, S. D., and Rather, P. N. (2013). Streptomycin inhibits quorum sensing in Acinetobacter baumannii. Antimicrob. Agents Chemother. 57, 1926-1929. doi: 10.1128/AAC.02161-12

Servin, A. L. (2004). Antagonistic activities of lactobacilli and bifidobacteria against microbial pathogens. FEMS Microbiol. Rev. 28, 405-440. doi: 10.1016/j.femsre.2004.01.003

Sierig, G., Cywes, C., Wessels, M. R., and Ashbaugh, C. D. (2003). Cytotoxic effects of streptolysin $\mathrm{O}$ and streptolysin $\mathrm{S}$ enhance the virulence of poorly encapsulated group a streptococci. Infect. Immun. 71, 446-455. doi: 10.1128/IAI.71.1.446-455.2003

Sjolinder, H., Lovkvist, L., Plant, L., Eriksson, J., Aro, H., Jones, A., et al. (2008). The ScpC protease of Streptococcus pyogenes affects the outcome of sepsis in a murine model. Infect. Immun. 76, 3959-3966. doi: 10.1128/IAI.00128-08

Spurbeck, R. R., and Arvidson, C. G. (2010). Lactobacillus jensenii surface associated proteins inhibit Neisseria gonorrhoeae adherence to epithelial cells. Infect. Immun. 78, 3103-3111. doi: 10.1128/IAI.01200-09

Velraeds, M. M., van de Belt-Gritter, B., van der Mei, H. C., Reid, G., and Busscher, H. J. (1998). Interference in initial adhesion of uropathogenic bacteria and yeasts to silicone rubber by a Lactobacillus acidophilus biosurfactant. J. Med. Microbiol. 47, 1081-1085. doi: 10.1099/00222615-47-12-1081

Velraeds, M. M., van der Mei, H. C., Reid, G., and Busscher, H. J. (1996). Inhibition of initial adhesion of uropathogenic Enterococcus faecalis by biosurfactants from Lactobacillus isolates. Appl. Environ. Microbiol. 62, 1958-1963.

Vielfort, K., Sjolinder, H., Roos, S., Jonsson, H., and Aro, H. (2008). Adherence of clinically isolated lactobacilli to human cervical cells in competition with Neisseria gonorrhoeae. Microbes Infect. 10, 1325-1334. doi: 10.1016/j.micinf.2008.07.032

Zhang, Y. C., Zhang, L. W., Tuo, Y. F., Guo, C. F., Yi, H. X., Li, J. Y., et al. (2010). Inhibition of Shigella sonnei adherence to HT-29 cells by lactobacilli from Chinese fermented food and preliminary characterization of S-layer protein involvement. Res. Microbiol. 161, 667-672. doi: 10.1016/j.resmic.2010.06.005

Conflict of Interest Statement: The authors declare that the research was conducted in the absence of any commercial or financial relationships that could be construed as a potential conflict of interest.

Copyright (c) 2016 Saroj, Maudsdotter, Tavares and Jonsson. This is an open-access article distributed under the terms of the Creative Commons Attribution License (CC BY). The use, distribution or reproduction in other forums is permitted, provided the original author(s) or licensor are credited and that the original publication in this journal is cited, in accordance with accepted academic practice. No use, distribution or reproduction is permitted which does not comply with these terms. 